\title{
Normal families of meromorphic functions sharing values or functions
}

\author{
Yunbo Jiang ${ }^{*}$ and Zongsheng Gao
}

\author{
* Correspondence: jiangyunbo@ss. \\ buaa.edu.cn \\ School of Mathematics and \\ Systems Science and LMIB, Beihang \\ University, Beijing, 100191, People's \\ Republic of China
}

\begin{abstract}
In this paper, we investigate the normal families of meromorphic functions concerning shared values and shared analytic functions and prove some normal criteria that generalize or extend some results obtained by Q. C. Zhang, Y. T. Li and Y. X. Gu, J. M. Chang.

Mathematics Subject Classification (2000): 30D45; 30035.

Keywords: meromorphic function, holomorphic function, shared value, shared analytic function, L. Yang's inequality, normal family
\end{abstract}

\section{Introduction and main results}

The notations and concepts used in this paper can be found in [1-3]. In this paper, We also use $f(z)=a \Rightarrow g(z)=b$ to stand for $g(z)=b$ when $f(z)=a$.

Let $D$ be a domain in the complex plane $C, \mathcal{F}$ be a family of meromorphic functions defined in $D . \mathcal{F}$ is said to be normal in $D$, in the sense of Montel, if every sequence $f_{n}(z) \in \mathcal{F}(n=1,2, \ldots)$ has a subsequence $f_{n_{k}}(z)(k=1,2, \ldots)$ that converges spherically locally uniformly in $D$, to a meromorphic function or $\infty($ see $[2,4,5])$.

In 1998, Y. F. Wang and M. L. Fang [6] proved the following theorem.

Theorem A. Let $\mathcal{F}$ be a family of meromorphic functions in $D, n, k \in \mathbf{N}$ with $n \geq k$ +2 . If for every function $f \in \mathcal{F}, f$ has only zeros of order at least $n$ and $f^{(k)} \neq 1$, then $\mathcal{F}$ is normal in $D$.

In 2004, M. L. Fang and L. Zalcman [7] proved the following theorem.

Theorem B. Let $\mathcal{F}$ be a family of meromorphic functions in $D$, and $n$ be a positive integer. If for each pair of functions $f$ and $g$ in $\mathcal{F}, f$ and $g$ share the value 0 and $f^{n} f^{\prime}$ and $g^{n} g^{\prime}$ share a non-zero value $b$ in $D$, then $\mathcal{F}$ is normal in $D$.

In 2008, Q. C. Zhang [8] proved the following Theorems C and D, which generalized the condition $f^{(k)} \neq 1$ in Theorem A to shared value when $k=1$ and generalized Theorem $\mathrm{B}$ when $n \geq 2$, respectively.

Theorem $\mathrm{C}$. Let $\mathcal{F}$ be a family of meromorphic functions in $D$ satisfying that all of zeros and poles of $f \in \mathcal{F}$ have multiplicities at least 3. If for each pair of functions $f$ and $g$ in $\mathcal{F}, f$ and $g^{\prime}$ share a non-zero value $b$ in $D$, then $\mathcal{F}$ is normal in $D$.

Theorem D. Let $\mathcal{F}$ be a family of meromorphic functions in $D$, and $n$ be a positive integer. If $n \geq 2$ and for each pair of functions $f$ and $g$ in $\mathcal{F}, f^{n} f$ and $g^{n} g^{\prime}$ share a nonzero value $b$ in $D$, then $\mathcal{F}$ is normal in $D$.

(c) 2011 Jiang and Gao; licensee Springer. This is an Open Access article distributed under the terms of the Creative Commons Attribution License (http://creativecommons.org/licenses/by/2.0), which permits unrestricted use, distribution, and reproduction in any medium, provided the original work is properly cited. 
In 2009, Y. T. Li and Y. X. Gu [9] proved the following theorem with high order derivatives.

Theorem E. Let $\mathcal{F}$ be a family of meromorphic functions defined in a domain $D$. Let $k, n \geq k+2$ be positive integers and $a \neq 0$ be a finite complex number. If $\left(f^{n}\right)^{(k)}$ and $\left(g^{n}\right)^{(k)}$ share $a$ in $D$ for every pair of functions $f, g \in \mathcal{F}$, then $\mathcal{F}$ is normal in $D$.

In this paper, we investigate the normal family of meromorphic functions with higher order derivatives and obtain the following two theorems, which generalize Theorems C, D and E.

Theorem 1.1. Suppose that $d(\geq 0)$ is an integer, $p(z)$ is an analytic function in $D$, and the multiplicity of its all zeros is at most $d$. Let $\mathcal{F}$ be a family of holomorphic functions in $D$; the multiplicity of all zeros of $f \in \mathcal{F}$ is at least $k+d+2$. If for each pair of functions $f$ and $g$ in $\mathcal{F}, f^{(k)}$ and $g^{(k)}$ share $p(z)$ in $D$, then $\mathcal{F}$ is normal in $D$.

Theorem 1.2. Suppose that $d(\geq 0)$ is an integer, $p(z)$ is an analytic function in $D$, and the multiplicity of its all zeros is at most $d$. Let $\mathcal{F}$ be a family of meromorphic functions in $D$, the multiplicity of all zeros and poles of $f \in \mathcal{F}$ is at least $k+2 d+2$. If for each pair of functions $f$ and $g$ in $\mathcal{F}, f^{(k)}$ and $g^{(k)}$ share $p(z)$ in $D$, then $\mathcal{F}$ is normal in $D$.

Corollary 1.1. Let $\mathcal{F}$ be a family of meromorphic functions in $D$; the multiplicity of all zeros and poles of $f \in \mathcal{F}$ is at least $k+2 . a \neq 0$ is a finite complex number. If for each pair of functions $f$ and $g$ in $\mathcal{F}, f^{(k)}$ and $g^{(k)}$ share $a$ in $D$, then $\mathcal{F}$ is normal in $D$.

Corollary 1.2. Suppose that $d(\geq 0)$ is an integer, $p(z)$ is an analytic function in $D$, and the multiplicity of its all zeros is at most $d$. Let $\mathcal{F}$ be a family of holomorphic functions in $D, n$ be a positive integer. If $n \geq d+2$ and for each pair of functions $f$ and $g$ in $\mathcal{F}, f^{n} f$ and $g^{n} g^{\prime}$ share $p(z)$ in $D$, then $\mathcal{F}$ is normal in $D$.

Corollary 1.3. Suppose that $d(\geq 0)$ is an integer, $p(z)$ is an analytic function in $D$, and the multiplicity of its all zeros is at most $d$. Let $\mathcal{F}$ be a family of meromorphic functions in $D, n$ be a positive integer. If $n \geq 2 d+2$ and for each pair of functions $f$ and $g$ in $\mathcal{F}, f^{n} f^{\prime}$ and $g^{n} g^{\prime}$ share $p(z)$ in $D$, then $\mathcal{F}$ is normal in $D$.

Remark 1.1. From Corollary 1.1, we can deduce Theorems $C$ and E; from Corollary 1.3, we can deduce Theorem D.

About the normality concerning shared value of meromorphic functions with its derivatives, J. M. Chang [10] recently obtained the following theorem.

Theorem $\mathbf{F}$. Let $\mathcal{F}$ be a family of meromorphic functions in $D, a, b$ be two distinct finite non-zero complex numbers. If for every $f \in \mathcal{F}, f(z)=a \Rightarrow f^{\prime}(z)=a, f^{\prime}(z) \neq b$, and $f^{\prime \prime}(z) \neq b$, then $\mathcal{F}$ is normal in $D$.

Thus, a natural question is: whether $\mathcal{F}$ is normal if we replace the condition $f(z)=a$ $\Rightarrow f^{\prime}(z)=a$ in Theorem $\mathrm{F}$ by $f^{\prime}(z)=a \Rightarrow f(z)=a$. We answer this question by the following result.

Theorem 1.3. Let $\mathcal{F}$ be a family of meromorphic functions in $D, a, b$ be two distinct finite complex numbers. If for every $f \in \mathcal{F}, f^{\prime}(z)=a \Rightarrow f(z)=a, f^{\prime}(z) \neq b$, and all of its zeros have multiplicity at least 2 , then $\mathcal{F}$ is normal in $D$.

\section{Lemmas}

Lemma 2.1. Let $d(\geq 0), k(\geq 1)$ be two integers, $p(z)=a_{d} z^{d}+a_{d-1} z^{d-1}+\cdots+a_{1} z+a_{0}$ be a polynomial, where $a_{d}(\neq 0), a_{d-1}, \ldots, a_{0}$ are constants. If $g(z)$ is a non-constant polynomial, the multiplicity of its all zeros is at least $k+d+2$, then $g^{(k)}(z)-p(z)$ has at least two distinct zeros, and $g^{(k)}(z)-p(z) \neq 0$. 
Proof We discuss in two cases:

Case 1. If $g^{(k)}(z)-p(z) \neq 0$, then $g^{(k)}(z)-p(z) \equiv C$, where $C$ is a constant. So $g(z)=\frac{a_{d} z^{d+k}}{(d+k)(d+k-1) \cdots(d+1)}+\frac{a_{d-1} z^{d+k-1}}{(d+k-1)(d+k-2) \cdots d}+\cdots+\frac{a_{0} z^{k}}{k(k-1) \cdots 1}+q(z)$, where $q^{(k)}(z) \equiv C$, i.e., $q(z)$ is a polynomial of degree at most $k$, then $g(z)$ is a polynomial of degree $k+d$, which contradicts with that the multiplicity of all zeros of $g(z)$ is at least $k+d+2$.

Case 2. If $g^{(k)}(z)-p(z)$ has only one zero $\xi_{0}$, we assume $g^{(k)}(z)-p(z)=A\left(z-\xi_{0}\right)^{r}$, where $A$ is a non-zero constant, $r$ is a positive integer.

We discuss the following three cases:

(i) If $r<d+1$, then $g(z)$ is a non-constant polynomial of degree at most $k+d$, which contradicts with that the multiplicity of all zeros of $g(z)$ is at least $k+d+2$.

(ii) If $r=d+1$, then $g^{(k+d+1)}(z)=A \cdot r \cdot(r-1) \cdots \cdot 2 \cdot 1$, so $g^{(k+d+1)}(z)$ has no zero. Since the multiplicity of all zeros of $g(z)$ is at least $k+d+2$, then $g(z)$ has no zero, which contradicts with $g(z)$ is a non-constant polynomial.

(iii) If $r>d+1$, then $g^{(k+d)}(z)-a_{d} \cdot d \cdot(d-1) \cdots \cdot 2 \cdot 1=A \cdot r \cdot(r-1) \cdots \cdot(r-d+1)(z$ $\left.-\xi_{0}\right)^{r-d}, g^{(k+d+1)}(z)=A \cdot r \cdot(r-1) \cdots . .(r-d)\left(z-\xi_{0}\right)^{r-d-1}$, so $\xi_{0}$ is the unique zero of $g^{(k}$ ${ }^{+d+1)}(z)$. Since $g(z)$ is a non-constant polynomial and the multiplicity of all zeros of $g(z)$ is at least $k+d+2$, then $\xi_{0}$ is a zero of $g$, thus, $g^{(k+d)}\left(\xi_{0}\right)=0$, which contradicts with $g$ ${ }^{(k+d)}\left(\xi_{0}\right)=a_{d} \cdot d \cdot(d-1) \cdots \cdot 2 \cdot 1 \neq 0$.

From Case 1 and Case 2, we know $g^{(k)}(z)-p(z)$ has at least two distinct zeros.

If $g^{(k)}(z)-p(z) \equiv 0$, then similar to the proof of Case 1 , we obtain that $g(z)$ is a polynomial of degree $k+d$ and get a contradiction since that the multiplicity of all zeros of $g(z)$ is at least $k+d+2$. Then, $g^{(k)}(z)-p(z) \neq 0$.

Lemma 2.2. Let $d(\geq 0), k(\geq 1)$ be two integers, $p(z)=a_{d} z^{d}+a_{d-1} z^{d-1}+\cdots+a_{1} z+$ $a_{0}$ be a polynomial, where $a_{d}(\neq 0), a_{d-1}, \ldots, a_{0}$ are constants. If $g(z)$ is a rational function and not a polynomial, and the multiplicity of all the zeros and poles of $g(z)$ is at least $k+2 d+2$, then $g^{(k)}(z)-p(z)$ has at least two distinct zeros, and $g^{(k)}(z)-p(z) \neq 0$.

Proof Since $g(z)$ is a rational function and not a polynomial, then obviously $g^{(k)}(z)-p$ $(z) \equiv 0$. Let

$$
\begin{aligned}
g(z) & =B \frac{\left(z-\gamma_{1}\right)^{p_{1}}\left(z-\gamma_{2}\right)^{p_{2}} \cdots\left(z-\gamma_{n}\right)^{p_{n}}}{\left(z-\delta_{1}\right)^{q_{1}}\left(z-\delta_{2}\right)^{q_{2}} \cdots\left(z-\delta_{m}\right)^{q_{m}}} \\
p & =p_{1}+p_{2}+\cdots+p_{n}, \quad q=q_{1}+q_{2}+\cdots+q_{m},
\end{aligned}
$$

where $B$ is a non-zero constant, $\gamma_{i}(i=1,2, \ldots, n)$ and $\delta_{j}(j=1,2, \ldots, m)$ are the zeros and poles of $g(z)$, their multiplicity are $p_{i}(i=1,2, \ldots, n)$ and $q_{j}(j=1,2, \ldots, m)$, respectively. Then, $p_{i}, q_{j} \geq k+2 d+2(i=1,2, \ldots, n, j=1,2, \ldots, m)$.

Differentiating both sides of (2.1) step by step, we have

$$
g^{(k)}(z)=B \frac{\left(z-\gamma_{1}\right)^{p_{1}-k}\left(z-\gamma_{2}\right)^{p_{2}-k} \ldots\left(z-\gamma_{n}\right)^{p_{n}-k}}{\left(z-\delta_{1}\right)^{q_{1}+k}\left(z-\delta_{2}\right)^{q_{2}+k} \ldots\left(z-\delta_{m}\right)^{q_{m}+k}} h_{k}(z),
$$

where $h_{k}(z)=(p-q)(p-q-1) \cdots(p-q-k+1) z^{k(n+m-1)}+\cdots+c_{k}^{(1)} z+c_{k}^{(0)}$ is a polynomial, $c_{k}^{(i)}(i=0,1)$ are constants.

$$
g^{(k+d+1)}(z)=B \frac{\left(z-\gamma_{1}\right)^{p_{1}-k-d-1}\left(z-\gamma_{2}\right)^{p_{2}-k-d-1} \cdots\left(z-\gamma_{n}\right)^{p_{n}-k-d-1}}{\left(z-\delta_{1}\right)^{q_{1}+k+d+1}\left(z-\delta_{2}\right)^{q_{2}+k+d+1} \cdots\left(z-\delta_{m}\right)^{q_{m}+d+k+1}} h_{k+d+1}(z),
$$


where $h_{k+d+1}(z)=(p-q)(p-q-1) \cdots(p-q-k-d) z^{(k+d+1)(n+m-1)}+\cdots+c_{k+d+1}^{(1)} z+c_{k+d+1}^{(0)}$ is a polynomial, $c_{k+d+1}^{(i)}(i=0,1)$ are constants.

Next, we discuss in two cases.

Case 1. If $g^{(k)}(z)-p(z)$ has a unique zero $\xi_{0}$, then let

$$
g^{(k)}(z)-p(z)=\frac{D\left(z-\xi_{0}\right)^{l}}{\left(z-\delta_{1}\right)^{q_{1}+k}\left(z-\delta_{2}\right)^{q_{2}+k} \ldots\left(z-\delta_{m}\right)^{q_{m}+k}},
$$

where $D$ is a non-zero constant and $l$ is a positive integer.

Here, we discuss in two subcases.

Subcase 1.1. When $d \geq l$.

Differentiating both sides of (2.4), we have

$$
g^{(k+d+1)}(z)-p^{(d+1)}(z)=\frac{D \cdot R_{d+1}(z)}{\left(z-\delta_{1}\right)^{q_{1}+k+d+1}\left(z-\delta_{2}\right)^{q_{2}+k+d+1} \cdots\left(z-\delta_{m}\right)^{q_{m}+k+d+1}},
$$

where $\begin{gathered}R_{d+1}(z)=(l-q-m k)(l-q-m k-1) \cdots(l-q-m k-d) z^{(d+1) m-(d-l+1)}+ \\ r_{d+1}^{(d+1) m-(d-l+1)-1)} z^{(d+1) m-(d-l+1)-1}+\cdots+r_{d+1}^{(1)} z+r_{d+1}^{(0)}\end{gathered}$ is a polynomial, $r_{d+1}^{((d+1) m-(d-l+1)-1)}, \ldots, r_{d+1}^{(1)}, r_{d+1}^{(0)}$ are constants.

By (2.2) and (2.4), since $d \geq l$, then $\operatorname{deg} h_{k}(z)+p-n k=q+k m+d \leq k(m+n-1)+$ $p-n k$, so $p-q \geq k+d$. Observe the form of $h_{k}(z)$, then $\operatorname{deg} h_{k}(z)=k(m+n-1)$, as $\operatorname{deg} h_{k}(z)+p-n k=q+k m+d$, thus $p-q=k+d$. From (2.3) and (2.5), $p-n k-n d-$ $n \leq(d+1) m-(d-l+1)$. Since $p-n k-n d-n-(d+1) m \geq p-\frac{p(k+d+1)}{k+2 d+2}-\frac{q(d+1)}{k+2 d+2}=\frac{(k+d)(d+1)}{k+2 d+2}$, then $l-d \geq p-n k-n d$ $-n-(d+1) m+1 \geq 1$, it contradicts with $d \geq l$.

Subcase 1.2. When $d<l$.

Differentiating both sides of (2.4), we have

$$
g^{(k+d+1)}(z)-p^{(d+1)}(z)=\frac{\left(z-\xi_{0}\right)^{l-d-1} U_{d+1}(z)}{\left(z-\delta_{1}\right)^{q_{1}+k+d+1}\left(z-\delta_{2}\right)^{q_{2}+k+d+1} \cdots\left(z-\delta_{m}\right)^{q_{m}+k+d+1}},
$$

where $U_{d+1}(z)=D(l-q-m k)(l-q-m k-1) \cdots(l-q-m k-d) z^{(d+1) m}+\cdots+u_{d+1}^{(1)} z+u_{d+1}^{(0)}$ is a polynomial, $u_{d+1}^{(i)}(i=0,1)$ are constants.

Differentiating both sides of (2.4) step by step for $d$ times, we can get that $\xi_{0}$ is a zero of $g^{(k+d)}(z)-p^{(d)}(z)$, as $p^{(d)}(z) \neq 0$, then $\gamma_{i} \neq \xi_{0}(i=1,2, \ldots, n)$.

Here, we discuss in three subcases.

Subcase 1.2.1. When $l<q+k m+d$. Similar to the proof of Subcase 1.1, we get $p-q$ $=k+d$. By (2.3), (2.6), and $\gamma_{i} \neq \xi_{0}(i=1,2, \ldots, n),(d+1) m \geq p-n k-n d-n$, then

$$
p \leq n k+n d+n+(d+1) m \leq \frac{p(k+d+1)}{k+2 d+2}+\frac{q(d+1)}{k+2 d+2}<p,
$$

which is a contradiction.

Subcase 1.2.2. When $l=q+k m+d$. If $p>q$, from (2.3), (2.6), and $\gamma_{i} \neq \xi_{0}(i=1,2, \ldots$, $n)$, we get $(d+1) m \geq p$ - nk - nd - n, then $p \leq n k+n d+n+(d+1) m \leq \frac{p(k+d+1)}{k+2 d+2}+\frac{q(d+1)}{k+2 d+2}<p$, which is a contradiction. Thus, $p \leq$ $q$. Then, from (2.3), (2.6), and $\gamma_{i} \neq \xi_{0}(i=1,2, \ldots, n)$, we have $l-d-1 \leq(k+d+1)(n+$ $m$ - 1). By simple calculation, 


$$
q \leq n(k+d+1)+m(d+1)-(k+d)<\frac{p(k+d+1)}{k+2 d+2}+\frac{q(d+1)}{k+2 d+2} \leq q,
$$

which is a contradiction.

Subcase 1.2.3. When $l>q+k m+d$. If $p \leq q$, by (2.2) and (2.4), then $l \leq q+k m+d$, which is a contradiction. Thus, $p>q$. By (2.3), (2.6) and $\gamma_{i} \neq \xi_{0}(i=1,2, \ldots, n)$, we get (d $+1) m \geq p-n k-n d-n$, then $p \leq n k+n d+n+(d+1) m \leq \frac{p(k+d+1)}{k+2 d+2}+\frac{q(d+1)}{k+2 d+2}<p$, which is a contradiction.

Case 2. If $g^{(k)}(z)-p(z)$ has no zero. Then, $l=0$ in (2.4), similar discussion to Case 1, we get a contradiction.

By Case 1 and Case 2, $g^{(k)}(z)-p(z)$ has at least two distinct zeros.

Lemma 2.3 (see [11]). Let $g(z)$ be a transcendental meromorphic function of finite order on $C$, and let $p(z)$ be a polynomial, $p(z) \neq 0$. Suppose that all zeros of $g(z)$ have multiplicity at least $k+1$. Then, $g^{(k)}(z)-p(z)$ has infinitely many zeros.

Lemma 2.4 (see [12]). Let $\mathcal{F}$ be a family of meromorphic functions on the unit disk $\Delta$, such that all zeros of functions in $\mathcal{F}$ have multiplicity greater than or equal to $l$, and all poles of functions in $\mathcal{F}$ have multiplicity greater than or equal to $j$. Let $\alpha$ be a real number satisfying $-l<\alpha<j$. Then, $\mathcal{F}$ is not normal in any neighborhood of $z_{0} \in \Delta$, if and only if there exist

(i) points $z_{k} \in \Delta, z_{k} \rightarrow z_{0}$;

(ii) positive numbers $\rho_{k}, \rho_{k} \rightarrow 0$, and

(iii) functions $f_{k} \in \mathcal{F}$ such that $\rho_{k}^{\alpha} f_{k}\left(z_{k}+\rho_{k} \zeta\right) \rightarrow g(\zeta)$ spherically locally uniformly in C, where $g(\zeta)$ is a nonconstant meromorphic function. The function may be taken to satisfy the normalization $g^{\#}(z) \leq g^{\#}(0)=1(z \in C)$. Here, $g^{\#}(z)$ denotes the spherical derivative of $g(z)$.

Lemma 2.5 (see [13]). Suppose that $f(z)$ is a meromorphic function on $\mathbf{C}$, if the spherical derivative $f^{*}(z)$ of $f(z)$ is bounded, then the order of $f(z)$ is at most 2 .

Lemma 2.6 (see [14]). Suppose that $f(z)$ is a transcendental meromorphic on $\mathbf{C}, k$ is a positive integer. If $\varepsilon$ is a positive number, $a, b$ are two dinstinct finite complex numbers, then

$$
\begin{gathered}
T\left(r, f^{(k)}\right)<\left(1+\frac{1}{2 k}\right) N\left(r, \frac{1}{f^{(k)}-a}\right)+\left(1+\frac{1}{2 k}\right) N\left(r, \frac{1}{f^{(k)}-b}\right) \\
-N\left(r, \frac{1}{f^{(k+1)}}\right)+\varepsilon T\left(r, f^{(k)}\right)+S\left(r, f^{(k)}\right) .
\end{gathered}
$$

Lemma 2.7 (see [15]). Let $g$ be a meromorphic function with finite order on C. If $g$ has only finitely many critical values, then it has only finitely many asymptotic values.

Lemma 2.8 (see [16]). Let $f(z)$ be meromorphic on $\mathbf{C}$ such that the set of its finite critical and asymptotic values is bounded. Then, there exists a positive number $r_{0}$, such that if $|z|>r_{0}$ and $|f(z)|>r_{0}$, then

$$
\left|f^{\prime}(z)\right| \geq \frac{|f(z)| \log |f(z)|}{16 \pi|z|} .
$$

Lemma 2.9. Suppose that $f(z)$ is a meromorphic function of finite order on $\mathbf{C}, \boldsymbol{a}$ and $b$ are two dinstinct finite complex numbers. If $f^{\prime}(z)=a \Rightarrow f(z)=0$, and $f^{\prime}(z) \neq b$, then $f$ is a rational function. 
Proof Suppose that $f$ is not a rational function. Since $f$ is a meromorphic function of finite order, then $f^{\prime}$ is also a meromorphic function of finite order. Because $f^{\prime}(z) \neq b$, then from Lemma 2.6, $f^{\prime}-a$ has infinitely many zeros $\left\{z_{n}\right\}$, and $z_{n} \rightarrow \infty(n \rightarrow \infty)$. Since $f^{\prime}(z)=a \Rightarrow f(z)=0$, hence $f\left(z_{n}\right)=0$.

Let $F(z)=f(z)-b z$, then $F^{\prime}(z)=f^{\prime}(z)-b \neq 0$, i.e., $F$ has no finite critical value. By Lemmas 2.7 and 2.8 , we have

$$
\left|F^{\prime}\left(z_{n}\right)\right| \geq \frac{\left|F\left(z_{n}\right)\right| \log \left|F\left(z_{n}\right)\right|}{16 \pi\left|z_{n}\right|}
$$

By simple calculation, we have

$$
|a-b| \geq \frac{|b|\left|z_{n}\right| \log \left|b z_{n}\right|}{16 \pi\left|z_{n}\right|} \rightarrow \infty(n \rightarrow \infty),
$$

which is a contradiction. Then, $f$ is a rational function.

Lemma 2.10 (see [6]). Let $f(z)=a_{n} z^{n}+a_{n-1} z^{n-1}+\cdots+a_{0}+\frac{q(z)}{p(z)}$, where $a_{0}, a_{1}, \ldots, a_{n}$ are constants with $a_{n} \neq 0, q(z)$ and $p(z)$ are two coprime polynomials with deg $q(z)<$ $\operatorname{deg} p(z), k$ be a positive integer. If $f^{(k)} \neq 1$, then we have

(i) $n=k$, and $k ! a_{k}=1$;

(ii) $f(z)=\frac{1}{k !} z^{k}+\cdots+a_{0}+\frac{1}{(a z+b)^{m}}$;

(iii) If the zeros of $f$ are of order $\geq k+1$, then $m=1$ in (ii) and $f(z)=\frac{(c z+d)^{k+1}}{a z+b}$, where $c(\neq 0), d$ are constants.

Lemma 2.11 (see $[6,17]$ ). Let $R$ be a rational function such that $R^{\prime} \neq 0$ on $\mathbf{C}$. Then, either $R=a z+b$ or $R=\frac{a}{\left(z+z_{0}\right)^{n}}+b$, where $a(\neq 0), b$ and $z_{0}$ are constants, and $n$ is a positive integer.

\section{Proof of Theorem 1.1}

For any point $z_{0}$ in $D$, either $p\left(z_{0}\right)=0$ or $p\left(z_{0}\right) \neq 0$.

Case 1. When $p\left(z_{0}\right)=0$. We may assume $z_{0}=0$. Then, $p(z)=a_{l} z^{l}+a_{l+1} z^{l+1}+\cdots=$ $z^{l} h(z)$, where $a_{l}(\neq 0), a_{l+1}, \cdots$ are constants, $l \geq 1$.

Let $M=\left\{G_{j} \mid G_{j}=\frac{f_{j}(z)}{z^{l}}, f_{j} \in \mathcal{F}\right\}$. If $M$ is not normal at 0 , then by Lemma 2.4, there exist points $z_{t} \rightarrow 0$, positive numbers $\rho_{t} \rightarrow 0$ and $G_{t} \in M$ such that $g_{t}(\zeta)=\rho_{t}^{-k} G_{t}\left(z_{t}+\rho_{t} \zeta\right) \rightarrow g(\zeta)$ spherically locally uniformly in $\mathbf{C}$, where $g(\zeta)$ is a nonconstant meromorphic function in $\mathbf{C}$, and $g^{\sharp}(\zeta) \leq 1$. Thus, the order of $g(\zeta)$ is at most 2 .

Here, we discuss in two cases.

Case 1.1. There exists a subsequence of $\frac{z_{t}}{\rho_{t}}$, we may still denote it as $\frac{z_{t}}{\rho_{t}}$ such that $\frac{z_{t}}{\rho_{t}} \rightarrow c, c$ is a finite complex number. Then,

$$
\phi_{t}(\zeta)=\frac{f_{t}\left(\rho_{t} \zeta\right)}{\rho_{t}^{l+k}}=\frac{\left(\rho_{t} \zeta\right)^{l} G_{t}\left(z_{t}+\rho_{t}\left(\zeta-\frac{z_{t}}{\rho_{t}}\right)\right)}{\left(\rho_{t}\right)^{l}\left(\rho_{t}\right)^{k}} \rightarrow \zeta^{l} g(\zeta-c)=H(\zeta)
$$

spherically locally uniformly in $\mathbf{C}$, so

$$
\phi_{t}^{(k)}(\zeta)-\frac{p\left(\rho_{t} \zeta\right)}{\rho_{t}^{l}}=\frac{f_{t}^{(k)}\left(\rho_{t} \zeta\right)-p\left(\rho_{t} \zeta\right)}{\rho_{t}^{l}} \rightarrow H^{(k)}(\zeta)-a_{l} \zeta^{l}
$$


spherically locally uniformly in $\mathbf{C}$.

Since $\forall f \in \mathcal{F}$, the multiplicity of all zeros of $f$ is at least $k+d+2$, the multiplicity of all zeros of $H$ is at least $k+d+2$, then from Lemmas 2.1 and $2.3 H^{(k)}(\zeta)-a_{l} \zeta \neq 0$, and $H^{(k)}(\zeta)-a_{l} \zeta^{l}$ has at least two distinct zeros.

Suppose that $\zeta_{1}, \zeta_{2}$ are two distinct zeros of $H^{(k)}(\zeta)-a_{l} \zeta$. We choose proper $\sigma>0$, such that $D\left(\zeta_{1}, \sigma\right) \cap D\left(\zeta_{2}, \sigma\right)=\varnothing$, where $D\left(\zeta_{1}, \sigma\right)=\left\{\zeta|| \zeta-\zeta_{1} \mid<\sigma\right\}, D\left(\zeta_{2}, \sigma\right)=\{\zeta|| \zeta$ $\left.\zeta_{2} \mid<\sigma\right\}$.

By Hurwitz's Theorem, there exists a subsequence of $f_{t}^{(k)}\left(\rho_{t} \zeta\right)-p\left(\rho_{t} \zeta\right)$, we may still denote it as $f_{t}^{(k)}\left(\rho_{t} \zeta\right)-p\left(\rho_{t} \zeta\right)$, then exists points $\zeta_{t}=\zeta_{1}$, and points $\tilde{\zeta}_{t}=\zeta_{2}$, such that when $t$ is large enough,

$$
f_{t}^{(k)}\left(\rho_{t} \zeta_{t}\right)-p\left(\rho_{t} \zeta_{t}\right)=0, f_{t}^{(k)}\left(\rho_{t} \tilde{\zeta}_{t}\right)-p\left(\rho_{t} \tilde{\zeta}_{t}\right)=0 .
$$

As for each pair of functions $f$ and $g$ in $\mathcal{F}, f^{(k)}$ and $g^{(k)}$ share $p(z)$ in $D$, then by (3.1) we can deduce that for any positive integer $r$, when $t$ is large enough,

$$
f_{r}^{(k)}\left(\rho_{t} \zeta_{t}\right)-p\left(\rho_{t} \zeta_{t}\right)=0, f_{r}^{(k)}\left(\rho_{t} \tilde{\zeta}_{t}\right)-p\left(\rho_{t} \tilde{\zeta}_{t}\right)=0 .
$$

For fixed $r$, let $t$ converges to $\infty$ in (3.2), then $\rho_{t} \zeta_{t} \rightarrow 0, \rho_{t} \tilde{\zeta}_{t} \rightarrow 0$, thus

$$
f_{r}^{(k)}(0)-p(0)=0
$$

Then, by the isolation property of zeros of $f_{r}^{(k)}(\zeta)-p(\zeta)$, when $t$ is large enough,

$$
\rho_{t} \zeta_{t}=\rho_{t} \tilde{\zeta}_{t}=0
$$

Thus, when $t$ is large enough, $\zeta_{1}=\zeta_{2}=0$, which contradicts with $D\left(\zeta_{1}, \sigma\right) \cap\left(\zeta_{2}, \sigma\right)=$ $\varnothing$. Thus, $M$ is normal at 0 .

Case 1.2. There exists a subsequence of $\frac{z_{t}}{\rho_{t}}$; we may still denote it as $\frac{z_{t}}{\rho_{t}}$ such that $\frac{z_{t}}{\rho_{t}} \rightarrow \infty$. Then,

$$
\begin{aligned}
f_{t}^{(k)}\left(z_{t}+\rho_{t} \zeta\right) & =\left(z_{t}+\rho_{t} \zeta\right)^{l} G_{t}^{(k)}\left(z_{t}+\rho_{t} \zeta\right)+\sum_{i=1}^{k} c_{i}\left(z_{t}+\rho_{t} \zeta\right)^{l-i} G_{t}^{(k-i)}\left(z_{t}+\rho_{t} \zeta\right) \\
& =\left(z_{t}+\rho_{t} \zeta\right)^{l} g_{t}^{(k)}(\zeta)+\sum_{i=1}^{k} c_{i}\left(z_{t}+\rho_{t} \zeta\right)^{l-i} \rho_{t}^{i} g_{t}^{(k-i)}(\zeta)
\end{aligned}
$$

where $c_{i}=l(l-1) \cdots(l-i+1) C_{k}^{l}$ when $l \geq i$, and $c_{i}=0$ when $l<i$.

Thus, we have

$$
\frac{a_{l} f_{t}^{(k)}\left(z_{t}+\rho_{t} \zeta\right)}{p\left(z_{t}+\rho_{t} \zeta\right)}-a_{l}=\left(g_{t}^{(k)}(\zeta)+\sum_{i=1}^{k} c_{i} \frac{g_{t}^{(k-i)}(\zeta)}{\left(\frac{z_{t}}{\rho_{t}}+\zeta\right)^{i}}\right) \frac{a_{l}}{h\left(z_{t}+\rho_{t} \zeta\right)}-a_{l} \rightarrow g^{(k)}(\zeta)-a_{l}
$$

spherically locally uniformly in $\mathbf{C}-\{\zeta \mid g(\zeta)=\infty\}$.

Since $\forall f \in \mathcal{F}$, the multiplicity of all zeros of $f$ is at least $k+d+2$, the multiplicity of all zeros of $g$ is at least $k+2$. Thus, by Lemmas 2.1 and 2.3, $g^{(k)}(\zeta)-a_{l}$ has at least two distinct zeros, and $g^{(k)}(\zeta)-a_{l} \equiv 0$. Suppose that $\zeta_{3}, \zeta_{4}$ are two distinct zeros of $g^{(k)}(\zeta)$ $a_{l}$. We choose proper $\sigma>0$, such that $D\left(\zeta_{3}, \sigma\right) \cap D\left(\zeta_{4}, \sigma\right)=\varnothing$, where $D\left(\zeta_{3}, \sigma\right)=\{\zeta|| \zeta$ $\left.-\zeta_{3} \mid<\sigma\right\}, D\left(\zeta_{4}, \sigma\right)=\left\{\zeta|| \zeta-\zeta_{4} \mid<\sigma\right\}$. 
By Hurwitz's Theorem, there exists a subsequence of $a_{l} f_{t}^{(k)}\left(z_{t}+\rho_{t} \zeta\right)-a_{l} p\left(z_{t}+\rho_{t} \zeta\right)$, we may still denote it as $a_{l} f_{t}^{(k)}\left(z_{t}+\rho_{t} \zeta\right)-a_{l} p\left(z_{t}+\rho_{t} \zeta\right)$, then exists points $\widehat{\zeta_{t}}=\zeta_{3}$, and points $\zeta_{t}^{*}=\zeta_{4}$, such that when $t$ is large enough,

$$
a_{l} f_{t}^{(k)}\left(z_{t}+\rho_{t} \hat{\zeta}_{t}\right)-a_{l} p\left(z_{t}+\rho_{t} \hat{\zeta}_{t}\right)=0, a_{l} f_{t}^{(k)}\left(z_{t}+\rho_{t} \zeta_{t}^{*}\right)-a_{l} p\left(z_{t}+\rho_{t} \zeta_{t}^{*}\right)=0
$$

Similar to the proof of Case 1.1, we get a contradiction. Then, $M$ is normal at 0 .

From Cases 1.1 and 1.2, we know $M$ is normal at 0 ; there exists $\Delta_{\rho}=\{z:|z|<\rho\}$ and a subsequence $G_{j_{k}}$ of $G_{j}$, such that $G_{j_{k}}$ converges spherically locally uniformly to a meromorphic function $G(z)$ or $\infty(k \rightarrow \infty)$ in $\Delta_{\rho}$.

Here, we discuss in two cases:

Case i. When $k$ is large enough, $f_{j_{k}}(0) \neq 0$. Then, $G(0)=\infty$. Thus, for $\forall$ constant $R>$ $0, \exists \sigma \in(0, \rho)$, we have $|G(z)|>R$ when $z \in \Delta_{\sigma}$. Thus, for sufficiently large $k,\left|G_{j_{k}}(z)\right|>\frac{R}{2}, \frac{1}{f_{j_{k}}}$ is holomorphic in $\Delta_{\sigma}$. Hence when $|z|=\frac{\sigma}{2}$,

$$
\left|\frac{1}{f_{j_{k}}(z)}\right|=\left|\frac{1}{G_{j_{k}}(z) z^{z}}\right| \leq \frac{2^{l+1}}{R \sigma^{l}} .
$$

By Maximum Principle and Montel's Theorem, $\mathcal{F}$ is normal at $z=0$.

Case ii. There exists a subsequence of $f_{j_{k}}$; we may still denote it as $f_{j_{k}}$ such that $f_{j_{k}}(0)=0$. Since $\forall f \in \mathcal{F}$, the multiplicity of all zeros of $f$ is at least $k+d+2$, then $G(0)$ $=0$. Thus, there exists $0<r<\rho$ such that $G(z)$ is holomorphic in $\Delta_{r}=\{z:|z|<r\}$ and has a unique zero $z=0$ in $\Delta_{r}$. Then, $G_{j_{k}}$ converges spherically locally uniformly to a holomorphic function $G(z)$ in $\Delta_{r} ; f_{j_{k}}$ converges spherically locally uniformly to a holomorphic function $z^{l} G(z)$ in $\Delta_{r}$. Hence $\mathcal{F}$ is normal at $z=0$.

By Case i and Case ii, $\mathcal{F}$ is normal at 0 .

Case 2. When $p\left(z_{0}\right) \neq 0$.

Suppose that $\mathcal{F}$ is not normal at $z_{0}$. By Lemma 2.4, there exist points $z_{n} \rightarrow z_{0}, \rho_{n} \rightarrow$ $0, f_{n} \in \mathcal{F}$ such that $\rho_{n}^{-k} f_{n}\left(z_{n}+\rho_{n} \zeta\right) \rightarrow g(\zeta)$ spherically locally uniformly in $\mathbf{C}, g(\zeta)$ is a non-constant meromorphic function in $\mathbf{C}$, and $g^{\#}(\zeta) \leq 1$.

By Lemma 2.5, $g(\zeta)$ is a meromorphic function of finite order, the multiplicity of all zeros of $f$ is at least $k+d+2$ for $\forall f \in \mathcal{F}$, thus the multiplicity of all zeros of $g$ is at least $k+d+2$.

Hence by Lemmas 2.1 and 2.3, $g^{(k)}(\zeta)-p\left(z_{0}\right)$ has at least two distinct zeros, and $g^{(k)}(\zeta)$ - $p\left(z_{0}\right) \neq 0$. Similar to the proof of Case 1.1, we get a contradiction. Thus, $\mathcal{F}$ is normal at $z_{0}$.

Thus, $\mathcal{F}$ is normal in $D$ as $z_{0}$ is arbitrary. The proof is complete.

\section{Proof of Theorem 1.2}

Similar to the proof of Theorem 1.1 and utilize Lemma 2.2, we can prove Theorem 1.2 immediately.

\section{Proof of Theorem 1.3}

Suppose that $\mathcal{F}$ is not normal in $D$, then there exists $z_{0} \in D$, such that $\mathcal{F}$ is not normal at $z_{0}$. Without loss of generality, we may assume $z_{0}=0$. By Lemma 2.4, there exist $z_{k} \rightarrow z_{0}, \rho_{k} \rightarrow 0, f_{k} \in \mathcal{F}$ such that $\rho_{k}^{-1} f_{k}\left(z_{k}+\rho_{k} z\right) \rightarrow R(z)$ spherically locally uniformly 
in $\mathbf{C}, R(z)$ is a non-constant meromorphic function in $\mathbf{C}$, and $R^{\#}(z) \leq 1$. Thus, $f_{k}^{\prime}\left(z_{k}+\rho_{k} z\right) \rightarrow R^{\prime}(z)$ spherically locally uniformly in $\mathbf{C}$.

By Hurwitz's Theorem, we can easily get: (i) $R^{\prime}=a \Rightarrow R=0$; (ii) $R^{\prime} \neq b$; (iii) the multiplicity of zeros of $R$ is at least 2 .

By Lemma 2.5, $R(z)$ is a meromorphic function of finite order. Moreover from (i), (ii), and Lemma $2.9, R$ is a rational function.

Here, we discuss in two cases:

Case 1. When $b=0$. Because $R^{\prime} \neq b$, by Lemma 2.11, $R=c z+d$ or $R=\frac{c}{\left(z+z_{0}\right)^{n}}+d$, where $c(\neq 0), d, z_{0}$ are constants, and $n$ is a positive integer. From (iii), we have $R=\frac{c}{\left(z+z_{0}\right)^{n}}+d$. If $a \neq 0$, then the roots number of $R^{\prime}=\frac{-n c}{\left(z+z_{0}\right)^{n+1}}=a$ is $n+1$, and the roots are different from each other, meanwhile the roots number of $R=0$ is at most $n$, it contradicts with $R^{\prime}=a \Rightarrow R=0$. Then, $a=0$, which contradicts with that $a$ and $b$ are distinct constants.

Case 2. When $b \neq 0$.

Here, we discuss in three subcases:

Subcase 2.1. If $R$ is a polynomial. From $R^{\prime} \neq b$ and Lemma 2.11, we have $R=c z+d$, where $c(\neq 0), d(\neq b)$ are constants, which contradicts with (iii).

Subcase 2.2. If $R=\frac{q(z)}{p(z)}$, where $q(z)$ and $p(z)$ are coprime polynomials, $\operatorname{deg} q(z)<\operatorname{deg}$ $p(z)$, then $R^{\prime}=\frac{q^{\prime} p-q p^{\prime}}{p^{2}}$. Since $\operatorname{deg} q(z)<\operatorname{deg} p(z)$, so $\operatorname{deg}\left(q^{\prime} p-q p^{\prime}\right)<\operatorname{deg}\left(p^{2}\right)$. Thus, 0 is the only one deficiency value of $R^{\prime}$, which contradicts with $R^{\prime} \neq b, b \neq 0$.

Subcase 2.3. If $R=a_{n} z^{n}+a_{n-1} z^{n-1}+\cdots+a_{0}+\frac{q(z)}{p(z)}$, where $a_{0}, a_{1}, \ldots, a_{n}$ are constants, $a_{n} \neq 0, q(z)$ and $p(z)$ are coprime polynomials, $\operatorname{deg} q(z)<\operatorname{deg} p(z)$. From Lemma 2.10 and (iii), $R=b z+a_{0}+\frac{1}{c z+d}=\frac{(l z+t)^{2}}{c z+d}$, where $c(\neq 0), d, l(\neq 0), t$ are constants. Since $a$ and $b$ are distinct, then the roots of $R^{\prime}-a=b-a-\frac{c}{(c z+d)^{2}}=0$ are two distinct complex numbers, meanwhile $R=0$ has a single root $z=-\frac{t}{l}$, which contradicts with $R^{\prime}=a \Rightarrow R$ $=0$.

Then, $\mathcal{F}$ is normal in $D$. The proof is complete.

\section{Acknowledgements}

The authors would like to thank the referees for their detailed and valuable comments. The work was supported by the National Natural Science Foundation of China(Grant No. 11171013) and the Fundamental Research Funds for the Central Universities.

\section{Authors' contributions}

YBJ and ZSG performed the proof and drafted the manuscript. All authors read and approved the final manuscript.

\section{Competing interests}

The authors declare that they have no competing interests.

Received: 27 March 2011 Accepted: 27 September 2011 Published: 27 September 2011

\section{References}

Hayman, WK: Meromorphic Functions. Clarendon Press, Oxford (1964)

Yang, L: Value Distribution Theory. Springer, Berlin (1993)

Yang, CC, Yi, HX: Uniqueness Theory of Meromorphic Functions. Kluwer, Dordrecht (2003)

Schiff, J: Normal Families. Springer, Berlin (1993)

Gu, YX, Pang, XC, Fang, ML: Theory of Normal Families and Its Application. Science Press, Beijing (2007)

6. Wang, YF, Fang, ML: Picard values and normal families of meromorphic functions with multiple zeros. Acta Math Sinica New Series. 14, 17-26 (1998). doi:10.1007/BF02563879

7. Fang, ML, Zalcman, L: A note on normality and shared values. J Aust Math Soc. 76, 141-150 (2004). doi:10.1017/ S1446788700008752 
8. Zhang, QC: Some normality criteria of meromorphic functions. Complex Var Elliptic Equ. 53, 791-795 (2008). doi:10.1080/17476930802124666

9. Li, YT, Gu, YX: On normal families of meromorphic functions. J Math Anal Appl. 354, 421-425 (2009). doi:10.1016/j. jmaa.2009.01.011

10. Chang, JM: Normality concerning shared values. Sci China Ser A. 52, 1717-1722 (2009). doi:10.1007/s11425-008-0172-2

11. Pang, XC, Yang, DG, Zalcman, L: Normal families of meromorphic functions whose derivatives omit a function. Comput Methods Funct Theory. 2, 257-265 (2002)

12. Zalcman, L: Normal families: new perspectives. Bull Amer Math Soc. 35, 215-230 (1998). doi:10.1090/S0273-0979-98$00755-1$

13. Clunie, J, Hayman, WK: The spherical derivative of integral and meromorphic functions. Comment Math Helv. 40, 117-148 (1966)

14. Yang, L: Precise fundamental inequalities and sum of deficiencies. Sci China Ser A. 34, 157-165 (1991)

15. Bergweiler, $W$, Eremenko, A: On the singularities of the inverse to a meromorphic function of finite order. Rev Mat Iberoamericana. 11, 355-373 (1995)

16. Rippon, PJ, Stallard, GM: Iteration of a class of hyperbolic meromorphic functions. Proc Amer Math Soc. 127, 3251-3258 (1999). doi:10.1090/50002-9939-99-04942-4

17. Changm, JM, Zalcman, L: Meromorphic functions that share a set with their derivatives. J Math Anal Appl. 338, 1020-1028 (2008). doi:10.1016/j.jmaa.2007.05.079

doi:10.1186/1029-242X-2011-72

Cite this article as: Jiang and Gao: Normal families of meromorphic functions sharing values or functions. Journal of Inequalities and Applications 2011 2011:72.

\section{Submit your manuscript to a SpringerOpen ${ }^{\odot}$} journal and benefit from:

- Convenient online submission

- Rigorous peer review

- Immediate publication on acceptance

- Open access: articles freely available online

- High visibility within the field

Retaining the copyright to your article

Submit your next manuscript at $\gg$ springeropen.com 